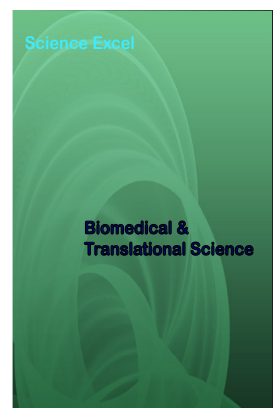

Correspondence

Lawrence M. Agius

27 "BALLARAT", GUZEPPE CARUANA STREET, TAL-VIRTU, RABAT, RBT09, MALTA, EUROPE

E-mail: lawrence.agius@um.edu.mt Tel: 356-21451752

- Received Date: 05 Jan 2021

- Accepted Date: 24 Apr 2021

- Publication Date: 01 May 2021

Copyright

(c) 2021 Science Excel. This is an openaccess article distributed under the terms of the Creative Commons Attribution 4.0 International license.

\title{
Predetermined Interplay of the Projected Malignant Transformation Step as Injury Non- Resolution
}

\author{
Lawrence M Agius \\ Department of Pathology, Mater Dei Hospital, Tal-Qroqa, University Of Malta Medical School, Msida, Malta, Europe
}

\begin{abstract}
Non-resolution of carcinogenesis is a paramount consideration in the systems of non-immunogenicity of tumor cells in general and as further projected by the infiltrating glioma cell within the brain parenchyma. In such terms, the performance dynamics for further progression are change attributes that bespeak of the systems of overlap and of interactivity of multiple pathways of pro-apoptosis and of anti-apoptosis as projected by mirrored system biology of cell component injury. It is within the single transformation phenomenon step in malignancy emergence that the pro-apoptosis projects the predetermination for non-resolution of cell component injury and as further determined by non-immunogenicity of the infiltrating glioma cells..
\end{abstract}

\section{Introduction}

Cell death pathways as mechanisms in the attempted control of tumorigenesis constitute a confirmation of events within embryonic and immunologic pathways of constitutive formalization as dictated by the resultant infiltrating glioma cells within the brain parenchyma. New cancer therapies may trigger tumor necrosis and an invivo method for apoptosis detection and the early treatment response would be clinically significant; taurine [1] H magnetic resonance spectroscopy signal in gliomas may be a robust apoptotic biomarker indicative of tumor necrosis [1]. In such terms, the overall balance of pro-apoptotic and anti-apoptotic pathways is significant as equilibrating systems of biologic measures in oncogenesis per se. It is further to such considerations that apoptosis and death of malignant cells are paradoxically results of attempts at the formulation of schemes for preservation of normal parenchymal survival of CNS function and structure. Comparative genomic hybridization confirms a pattern for anaplastic oligodendrogliomas as three disjunct genetically defined subgroups of oligodendroglioma [2]. SSFA2 gene mechanism of action inhibits cell proliferation and promotes apoptosis in gliomas [3].

\section{Cell Death Pathways}

The significant degrees of control of cell death pathways are incremental dimensions that bespeak for the introduction of measures in the face of multi-pathway progression in tumorigenesis. 5-aminolevulinic acid -sonodynamic therapy with high-intensity focused ultrasound may constitute tumor specific therapy for both the tumor mass and also for infiltrating glioma cells [4].

The distinctive caspase cascades and the equally effective anti-apoptosis systems participate within the realized outgoing effects of a process of malignant transformation within the verifiable dimensions for further progression of carcinogenesis. The formulation of balancing pathways provoking apoptosis are significant in terms of the process for constitutive preservation of non-injury as defined by tissue and organ homeostasis and only secondarily as cell preservation of the life span processes of cell formulas of survival. It is in such terms that the pronounced derivation of injury to cell components and DNA perpetuates the control of homeostatic mechanisms of antigenic identity of pathways for resolute survival of pathways of progression.

It is further to such identification of antigenic identity that the final resolution of initial stages of apoptosis are pathway determination as projected by ligand/receptor binding in the first instance. MicroRNAs are aberrantly expressed in various cancers and glioma and Mir-16-5 is frequently downregulated in astrocytomas and modulates proliferation, apoptosis and response to cytotoxic therapy [5]. Cycloartenold induces anti-proliferation on Glioma U87 cells via 
induction of cell cycle arrest and p38 MAPK-mediated apoptosis [6]. Such terms of resolution demand a formula for progression that is conveyed paradoxically by the carcinogenesis complex of interactivities and further constitutional determination by pro-apoptotic and anti-apoptotic pathways of non-resolution.

\section{Identifiable Parameters of Progression}

Proportional identification of injury to components of a cell are identification clues to a paramount determination as conveyed by systems of progression of such cell component injury. The emergence of such realization is pathway progression of the pro-apoptosis within schemes of attempted anti-apoptosis.

The further predetermination of cell injury is within schemes of projection as determined by antigenicity, on the one hand, and of receptor confirmation of binding of such antigens as indeed determined by formulas of injury progression in cells undergoing apoptosis.

\section{Dyshomeostasis}

TThe complex dyshomeostasis as confirmed and further realized in cells undergoing apoptosis is pathway delineation that extensively coordinates the outcome projection of systems for progression. Long non-coding RNA plays a crucial role in the progression of glioma and promotes glycolysis by regulating miR-152-3p/SLC2A1 axis in glioma [7]. The identity constitution of pathways of non-resolution of pro-apoptotic pathways is characterization of the essential features of the malignant change that transforms normal glial cells to infiltrating glioma cells. Compound $6 \mathrm{~d}$ (ursolic acid derivative) promotes apoptosis in glioma cells through down-regulation of cAMP [8]. Such measures within systems for progression are integral determinants of the malignant transformation as borne out by numerous cell types and within systems of essential non-resolution of cell component injury.

The apoptosis of such transforming cells is further projected as confirmatory systems predisposing to injury to DNA and to immunologic components of identification and realization. Photodynamic therapy with hematoporphyrin significantly increases cell apoptosis, induces the content of reactive oxygen species and decreases the mitochondrial membrane potential and thus indicating induced apoptosis [9]. The proportional dispersal of the injurious agent within cell component biologic mechanisms is a further determination of progression of the apoptosis that identifies and further confirms the projection of the malignant transformation process beyond ligandreceptor binding of such components as CD95 and ApoL ligands to their constitutive receptors. Gpx 4 is implicated in the proliferation, migration and apoptosis of glioma cells [10]. In terms therefore of identifiable constitution, the realization of injury to cells is itself a potent mechanistic determinant in a malignant transformation step in carcinogenic identification.

\section{Pro-Apoptosis and Anti-Apoptosis}

The significance of emergence of a balance between pro-apoptosis and anti-apoptosis is projected as system biology of multiple models for attempted resolution of a carcinogenesis within further system pathways of injury that in turn determines outcome dynamics for non-resolution of cell component injury. PSMB8 interference may inhibit the migration and proliferation of glioma cells by reducing expression of cyclin A, cyclin B1, cyclin D1, vimentin, and $\mathrm{N}$-cadherin, and by increasing expression of E-cadherin through modulation of ERK2/3 and PI3K/AKT signaling pathways [11]. The performance determinants of malignant transformation are system pathways that overlap with confirmatory realization as conveyed by the progressiveness of the malignant transformation step undergone by constitutive cells. It is within the further development of mechanistic pathways of such non-resolution that the emergence of tumorigenesis is both identifiable and further projected by systems of overlap and of redundancy as afforded by proapoptosis.

The significant identification of non-immunogenicity is conveyed by infiltrating glioma cells within the system biology of mirrored images as geometrically determined by the overall malignant lesion extending within the brain parenchyma.

\section{Performance Attributes}

Performance attributes significantly determine the identity of non-resolution as further predetermination of the proapoptosis as targeted within systems of biologic progression of the infiltrating glioma cell. Fibronectin 1 exerts a potent role in stimulating glioma growth, invasion and survival through the activation of the PI3K/AKT signaling pathway [12]. The atypical protein kinase RIOK3 contributes to glioma cell proliferation/survival, migration/invasion and the AKT/mTOR signaling pathway [13]. In such terms, the outcome dynamics for non-resolution are integral to a nonimmunogenicity that is identifiable determinant of injury nonresolution in its own right.

The performance hence of non-resolution as projected by progression of pro-apoptosis pathways is hence a significant contribution as portrayed within the pathways of simple predetermination as exerted by carcinogenic agents of multiidentity.

\section{Concluding Remarks}

The delivery of both pro-apoptosis and anti-apoptosis are signal pathways of consequential import, as identifiable by modification of systems of potential malignant transformation to an infiltrating glioma cells. It is such realization that affords the identification of a malignant transformation step as attribute determination of the infiltrating glioma cells. Proportional emergence is delivery confirmation within determinant systems of exchange and interactivity as cell component injury.

The emergence of a single step in malignant transformation is identifiable within the modeled system biology of such interactivity of pathway progression and interactivity as systems of projected potent predetermination of the constitutive glioma cells. The infiltrative phenotype is characterized as significant component biology that arises within effects projected by the non-immunogenicity of the infiltrating tumor cells and as further confirmed by dynamics of non-resolution.

\section{References}

1. COpstad KS, Bell BA, Griffiths JR, Howe FA “Taurine: a potential marker of apoptosis in gliomas” Br J Cancer 2009;100(5):789-94. 
2. Koschny R, Heidrun Holland H, Koschny T, Vitzthum H-E "Comparative genomic hybridisation pattern of non-anaplastic and anapaestic oligodendrogliomas - a meta-analysis" Pathol Res Pract 2006;202(1):23-30.

3. Zhu A, Li X, Wu H, Miao Z, Yuan F, Zhang F et al. "Molecular mechanism of SSFA2 deletion inhibiting cell proliferation and promoting cell apoptosis in glioma" Pathol Res Pract 2019;215(3): 600-606.

4. Suehiro S, Ohnishi T, Yamashita D, Kohno S, Inoue A, Nishikawa $M$ et al. "Enhancement of antitumor activity by using 5-ALA-mediated sonodynamic therapy to induce apoptosis in malignant gliomas: significance of high-intensity focused ultrasound on 5-ALA-SDT in a mouse glioma model" J Neurosurg 2018;129(6):1416-1428.

5. Krill A, Wolter M, Stojcheva N, Hertler C, Liesenberg F, Zapata $\mathrm{M}$ et al. "MiR-16-5p is frequently down-regulated in astrocytic gliomas and modulates glioma cell proliferation, apoptosis an response to cytotoxic therapy" Neuropathol Appl Neurobiol 2019;45(5):441-458.

6. Liu H, Li X, Yang A, Jin Z, Wang X, Wang Q et al.“Cycloartenol exerts anti-proliferative effects on Glioma U87 cells via induction of cell cycle arrest and p38 MAPK-mediated apoptosis" J BUON 2018;23(6):1840-1845.

7. She J, Zhang Y, Qin B, Wang Y, Zhu X "Long non-coding RNA LINC00174 promotes glycolysis and tumor progression by regulating miR-152-3p/SLC2A1 axis in glioma” J Exp Clin Cancer Res 2019;38(1):395.

8. Fan H, Geng L, Yang F, Dong X, He D, Zhang Y "Ursolic acid derivative induces apoptosis in glioma cells through downregulation of cAMP” Eur J Med Chem 2019;176:61-67.

9. Yuan S-X. Li J-L, Xu X-K, Chen W, Chen C, Kuang K-Q et al. "Underlying mechanism of the photodynamic activity of hematoporphyrin-induced apoptosis in U87 glioma cells" Int J Mol Med 2018;41(4): 2288-2296.

10. Zhao H, Ji B, Chen J, Huang Q, Lu X "Gpx $r$ is involved in the proliferation, migration and apoptosis of glioma cells" Pathol Res Pract 2017;213(6):626-633.

11. Yang B-Y, Song J-W, Sun H-Z, Xing J-C, Yang Z-H, Wei C-Y et al. "PSMB8 regulates glioma cell migration, proliferation, and apoptosis through modulating ERK1/2 and PI3K/AKT signaling pathways" boomed Pharmacother 2018;100:205-212.

12. Liao Y-X, Zhang Z-P, Zhao J, Liu J-P “Effects of fibronectin 1 on cell proliferation, senescence and apoptosis of human glioma cells through the PI3K/AKT signaling pathway" Cell Physiol Biochem 2018;48(3):1382-1396.

13. Zhang T. Ji D, Wang P, Liang D, Jin L, Shi $\mathrm{H}$ et al. “The atypical protein kinase RIOK3 contributes to glioma cell proliferation/ survival, migration/invasion and the AKT/mTOR signaling pathway" 